\title{
IGF-IR-dependent expression of Survivin is required for T-antigen-mediated protection from apoptosis and proliferation of neural progenitors
}

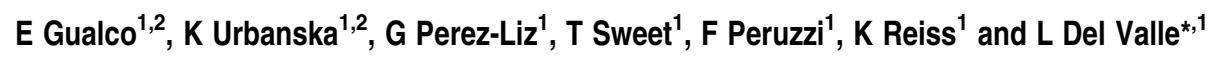

The insulin-like growth factor-1 receptor (IGF-IR) and the human polyomavirus JCV protein, T-antigen cooperate in the transformation of neuronal precursors in the cerebellum, which may be a contributing factor in the development of brain tumors. Because it is not clear why T-antigen requires IGF-IR for transformation, we investigated this process in neural progenitors from IGF-IR knockout embryos (ko-IGF-IR) and from their wild-type nontransgenic littermates (wt-IGF-IR). In contrast to wt-IGF-IR, the brain and dorsal root ganglia of ko-IGF-IR embryos showed low levels of the antiapoptotic protein Survivin, accompanied by elevated numbers of apoptotic neurons and an earlier differentiation phenotype. In wt-IGF-IR neural progenitors in vitro, induction of T-antigen expression tripled the expression of Survivin and accelerated cell proliferation. In ko-IGF-IR progenitors induction of T-antigen failed to increase Survivin, resulting in massive apoptosis. Importantly, ectopic expression of Survivin protected ko-IGF-IR progenitor cells from apoptosis and siRNA inhibition of Survivin activated apoptosis in wt-IGF-IR progenitors expressing T-antigen. Our results indicate that reactivation of the antiapoptotic Survivin may be a critical step in JCV T-antigen-induced transformation, which in neural progenitors requires IGF-IR.

Cell Death and Differentiation (2010) 17, 439-451; doi:10.1038/cdd.2009.146; published online 16 October 2009

The human neurotropic virus JCV, a member of the Polyomaviridae family, which also includes BKV and SV40, is the opportunistic etiological agent of the fatal demyelinating disease progressive multifocal leukoencephalopathy (PML). ${ }^{1}$ In addition to its function in the pathogenesis of PML, there is mounting evidence that links JCV with the development of cancer in humans. ${ }^{2}$ Human polyomaviruses have been shown to possess transforming abilities in vitro, induce brain tumors when inoculated into the brain of experimental animals and are suspected to participate in the process of oncogenesis in humans. ${ }^{2}$ A strong correlation has been established between the activation of the early viral genome and the development of a transformed phenotype. ${ }^{3}$ Polyomavirus-transforming antigens (T-antigens), encoded within the early genome, are the major suspects in the process of deregulating cellular homeostasis. ${ }^{4,5}$ Multiple interactions between T-antigen and cellular regulatory proteins have been detected at different levels, including signal transduction, gene expression, cellcycle progression, DNA damage and DNA repair mechanisms. ${ }^{6-10}$ Probably the best well-documented cellular targets for SV40 and JCV T-antigens are two major cell-cycle regulators, $\mathrm{p} 53$ and $\mathrm{pRb} .^{11,12}$ In addition, the necessity of the insulin-like growth factor-1 receptor (IGF-IR) in the process of cellular transformation induced by $\mathrm{T}$-antigen has been well established.
The first clues of the importance of IGF-IR in transformation were provided when mouse embryo fibroblasts (MEFs) isolated from IGF-IR knockout transgenic mice ( $\mathrm{R}^{-}$cells) failed to form colonies when exposed to SV40 T-antigen. ${ }^{13,14}$ Further experiments indicated that the signaling pathway used in the process of cellular transformation by T-antigen involves the tyrosine phosphorylation of insulin receptor substrate 1 (IRS-1) and the subsequent recruitment of $\mathrm{PI}-3$ kinase. ${ }^{15}$ In one report, however, the requirement of IGF-IR in the process of cellular transformation induced by T-antigen has been partially challenged. In that study, expression of SV40 T-antigen did not transform early passages of $R^{-}$cells, but instead lead to the development of anchorage independence and tumor formation by one late passage clone $\left(R^{-} 3 / T\right){ }^{16}$ Recently, we have shown that JCV T-antigen was also unable to transform MEFs lacking IGF-IR. ${ }^{17}$ Interestingly, MEFs expressing very low levels of IGF-IR (3000 molecules per cell) were refractory to transformation when exposed to T-antigen. The actual number of IGF-IR molecules that permitted T-antigen-induced transformation has been determined to be between 12000 and $22000 .^{17}$ In addition, we have shown that inhibition of the IGF-IR either by antisense strategies, ${ }^{18}$ dominant-negative IGF-IR mutant ${ }^{19}$ or by small molecular weight IGF-IR tyrosine kinase inhibitors ${ }^{20}$ compromised the survival of medulloblastoma cells in a T-antigen transgenic

\footnotetext{
${ }^{1}$ Department of Neuroscience, Center for Neurovirology, Temple University School of Medicine, Philadelphia, PA, USA

*Corresponding author: L Del Valle, Department of Neuroscience, Neuropathology Core \& Center for Neurovirology, 1900 North, 12th Street, Suite 244, Philadelphia, PA 19122, USA. Tel: (215) 204-0631; E-mail: luis.del.valle@ temple.edu

${ }^{2}$ These authors contributed equally to this work.

Keywords: Survivin; IGF-IR; JCV T-antigen; neurospheres; apoptosis; differentiation

Abbreviations: BrdU, 5-bromo 2-deoxyuridine; DRG, dorsal root ganglia; GalC, galactocerebroside; GFAP, glial fibrillary acidic protein; HRR, homologous recombination-dependent DNA repair; IAP, inhibitors of apoptosis; IGF-IR, insulin-like growth factor 1 receptor; mTOR, mammalian target of rapamycin; PML, progressive multifocal leukoencephalopathy; siRNA, small interfering RNA; TUNEL, terminal deoxynucleotidyl transferase dUTP nick end labeling

Received 19.3.09; revised 18.8.09; accepted 04.9.09; Edited by R De Maria; published online 16.10 .09
} 
mouse tumor model, further implicating IGF-IR in the process of transformation by JCV T-antigen.

Despite these multiple findings, it is not clear why T-antigen requires IGF-IR for transformation because the interactions between $\mathrm{T}$-antigen and $\mathrm{p} 53$ and pRb were not affected by the attenuation of IGF-IR tyrosine kinase activity. ${ }^{20}$ An additional clue to this mechanism has been provided by results from two independent studies involving a member of the inhibitors of apoptosis family, Survivin. This antiapoptotic protein is expressed at high levels during embryonic development, but its expression is completely silenced in adult and fully differentiated tissues. ${ }^{21}$ The first study showed that the transcriptional activation of Survivin depends on the activation of IGF-I/mTOR signaling pathway in prostate cancer cells. ${ }^{22}$ In the second study, a robust activation of Survivin was observed in JCV-infected cells in cases of PML, and this activation was corroborated in primary glial cell cultures infected in vitro with JCV. ${ }^{23}$ Although these two studies are based on different experimental models and involve different pathologies, they suggest that Survivin could represent a common link between JCV T-antigen and IGF-IR in both cellular transformation and in the inhibition of apoptosis, which eventually results in active viral replication and in the development of PML.

Therefore, we have now investigated early cellular responses to JCV T-antigen in neural progenitors from IGF-IR knockout embryos (ko-IGF-IR) and from wild-type nontransgenic littermates (wt-IGF-IR). Our results indicate that one of the mechanisms that could explain the necessity of IGF-IR in JCV T-antigen-mediated cellular transformation involves the reactivation of Survivin, which at least in neural progenitors requires the presence of functional IGF-IR.

\section{Results}

Histological and immunohistochemical characterization of the CNS in IGF-IR knockout mice. Previous phenotypical and morphological characterization of mouse embryos with targeted disruption of the IGF-IR gene showed alterations in the development of bone, muscle, skin and spinal cord. ${ }^{24}$ Our first set of experiments was aimed to show a detailed histological and immunohistochemical characterization of the brain and dorsal root ganglia (DRG) of IGF-IR knockout embryos (ko-IGF-IR) in comparison with their wild-type littermates (wt-IGF-IR).

Histological evaluation of the CNS revealed dramatic differences in development and differentiation of the brain and DRG. Although wild-type embryos possess larger brains with prominent flexures and proper neuronal layering, the brains of knockout embryos are considerably thinner and show smaller primitive neurons at the surface and a more populated germinal matrix, suggesting an earlier stage in migration (see montages and hematoxylin and eosin (H\&Es) in Figure 1). In addition, the DRG of wild-type embryos are larger and fully populated by well-differentiated neurons with abundant cytoplasm. As expected, the antiapoptotic protein Survivin is abundantly and robustly expressed in the brain and DRG of wild-type embryos, where no apoptotic cells are found by terminal deoxynucleotidyl transferase-mediated dUTP nick-end in situ labeling (TUNEL) assay. In contrast, knockout embryos show very weak expression of Survivin and abundant apoptotic cells can be detected in both the brain and DRG. Quantification of TUNEL-positive cells revealed $2 \%$ of cells undergoing apoptosis in the brain of wild-type mice compared with $37 \%$ in the knockout animals. In the DRG, $1.3 \%$ of cells are undergoing apoptosis in the wild type compared with $23 \%$ in the knockout embryos. The specificity of the Survivin pathway involvement in the inhibition of apoptosis was corroborated by immunohistochemical detection of other inhibitors of apoptosis family members, such as clAP1, which is not detectable in either knockout or wild-type brains (data not shown). In addition, levels of another prominent antiapoptotic protein, Bcl-2, were similar in both knockout and wild-type brains and DRG (Figure 1, lower panels).

In addition, there seems to be an inverse correlation between the expression of Survivin and the degree of development and differentiation of the CNS. Although the brains and DRG of wild-type mice, in which expression of Survivin is robust, show weak levels of the early marker Nestin and widespread expression of the late neuronal marker class III $\beta$-tubulin, their counterparts in the IGF-IR knockout mice show robust expression of Nestin and very low levels of $\beta$ IIItubulin, suggesting, along with the lack of layering, an earlier stage in the differentiation process. These changes are particularly noticeable in the DRG where large neurons with abundant cytoplasm in the wild-type embryos express robust $\beta$ III-tubulin, in contrast with the smaller and poorly differentiated neurons of the knockout where apoptotic cells are numerous and $\beta$ III-tubulin is barely expressed (Figure 1).

\section{Characterization of the IGF-IR knockout neural progenitors. Transgenic knockout embryos (embryonic day 16) were generated as a result of the breeding between IGF-IR knockout heterozygotes (IGF-IR ${ }^{+/-}$) (Figure 2a). Neural progenitors were isolated either from the knockout embryos or from age-matching wild-type (wt) nontransgenic littermates (IGF-IR ${ }^{+/+}$or IGF-IR ${ }^{+/-}$). Under growth-promoting culture conditions, both ko-IGF-IR and}

\footnotetext{
Figure 1 Immunohistochemical characterization of IGF-IR knockout mouse embryos. IGF-IR knockout embryos are smaller in size and volume than their wild-type littermates (montages). The brain of wild-type animals is considerably bigger, contains several flexures and is well layered, whereas knockout mice have thinner, flatter and less-differentiated brains. Although the brain and dorsal root ganglia (DRG) of wild-type animals contains bigger neurons with abundant cytoplasm, knockout mice contain smaller and more primitive neurons with scant cytoplasm (upper panels, hematoxylin and eosin). The antiapoptotic protein Survivin is expressed abundantly in the brain and DRG of wild-type mice, where no apoptosis is detected by TUNEL assay. IGF-IR knockout mice show very weak and low levels of Survivin and show numerous cells undergoing apoptosis. The levels of Survivin also correlate with the degree of differentiation. Although the early marker Nestin is detected in low levels in wild-type brain and DRG, its expression is robust in the smaller neurons of the knockout mice, in an inverse relation to the later neuronal marker class III $\beta$-tubulin, which is abundant and robust in larger neurons of wild-type brain and DRG, indicating a higher degree of differentiation. Other antiapoptotic pathways, including Bcl-2, showed no significant differences between the wild-type and knockout brains and DRG (lower panels). Original magnification for all brain panels $\times 200$. All DRG panels $\times 400$
} 

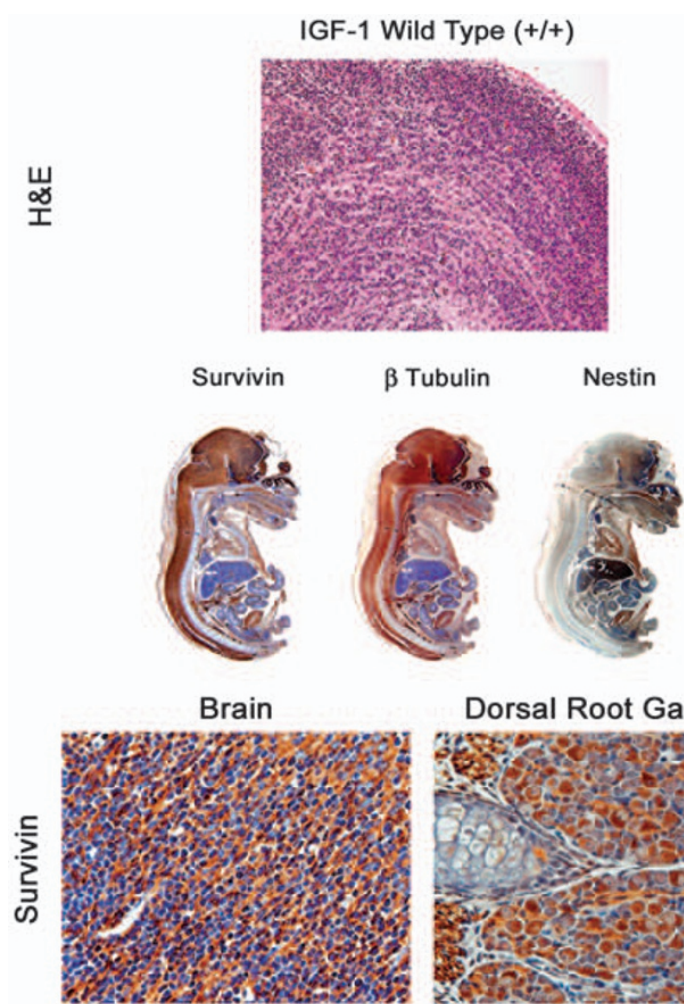

Dorsal Root Ganglia
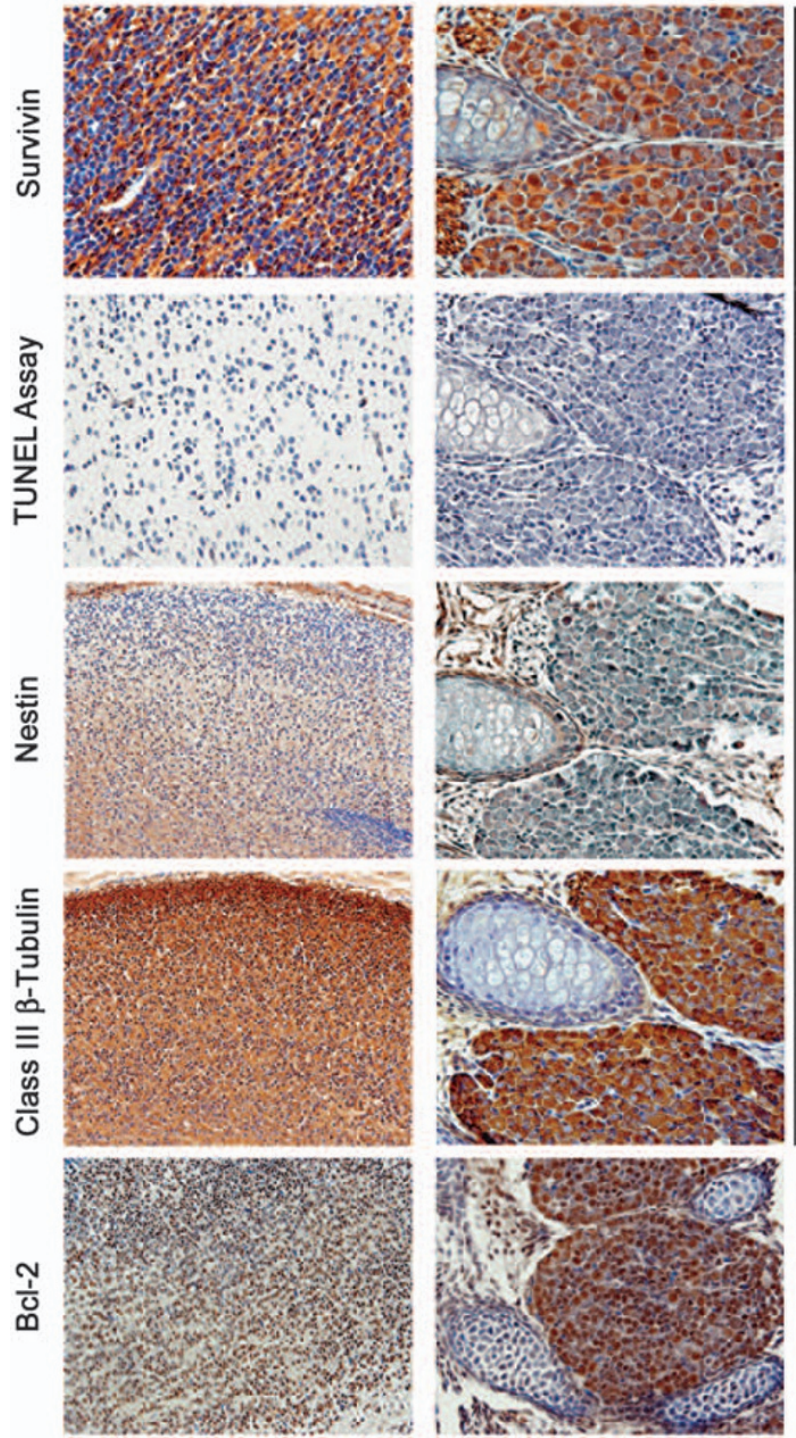

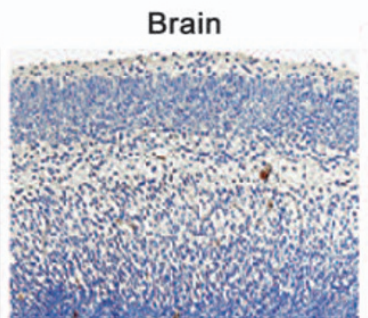

Dorsal Root Ganglia
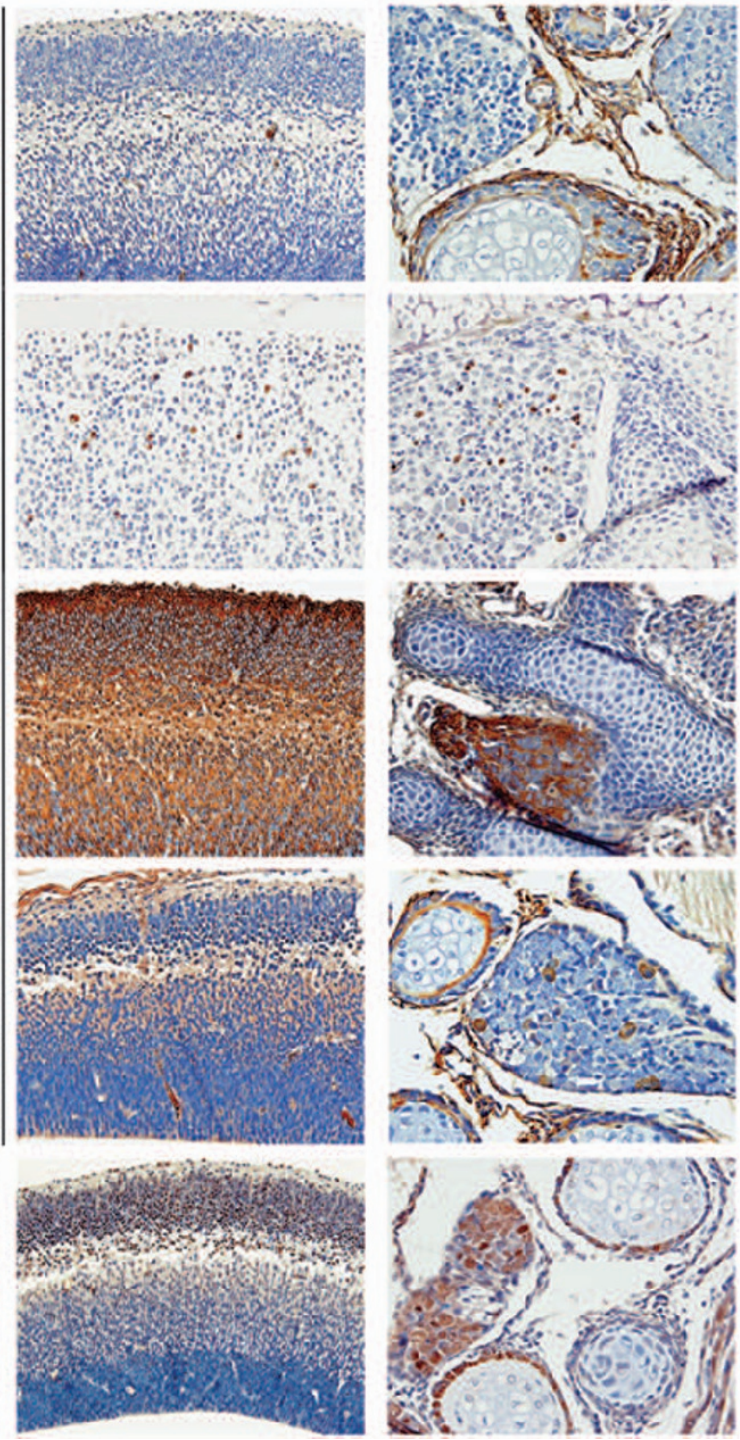


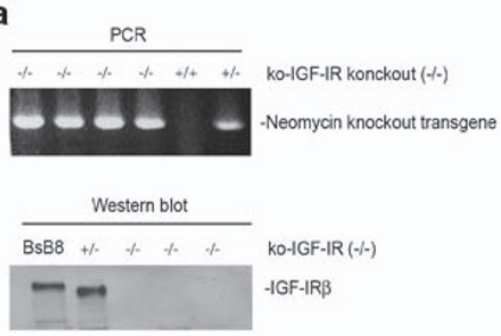

b

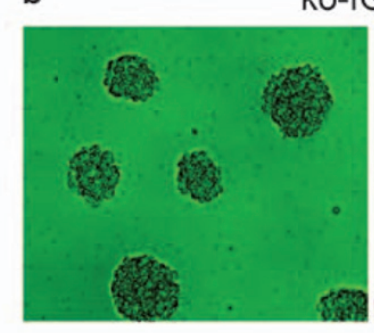

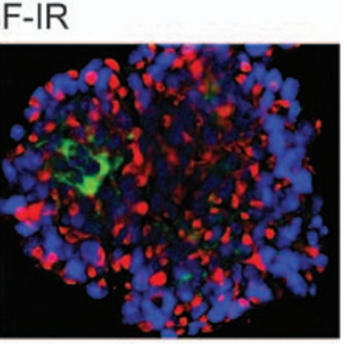

Nestin(red)/ $/$ III-Tubulin (green)
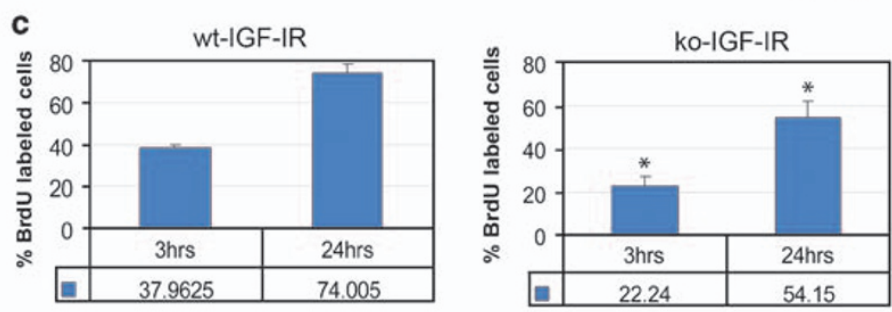

d
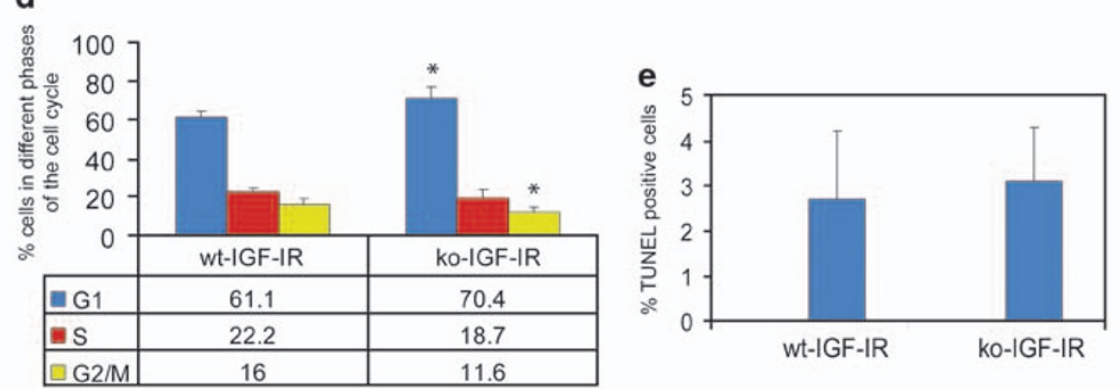

Figure 2 Characterization of neuronal progenitors from mouse embryos with targeted disruption of the IGF-IR gene (ko-IGF-IR). (a, upper) PCR detection of the knockout transgene used in targeted disruption of the IGF-IR gene. The embryos examined consist of four homozygotes $(-1-)$ (with both IGF-IR alleles disrupted), one heterozygote $(+I-)$ and one animal with the wild-type (wt) IGF-IR allele $(+I+)$; (a, lower) western blot analysis in which anti-IGF-IR antibody detected the IGF-IR $\beta$ subunit (95 kDa) in BsB8 cells (positive control) and in one IGF-IR knockout heterozygote $(+/-)$. The remaining three homozygotes $(-/-)$ are negative. (b) left) Twelve days culture of secondary neurospheres kept in cell growth-promoting culture. (b, right) Double labeling of proliferating secondary neurospheres with Nestin (rhodamine), and class III $\beta$-tubulin (fluorescein) showing cells committed toward a neuronal lineage. Note that only a few cells in the central portion of the proliferating neurosphere are positive for $\beta$ III-tubulin (green) and negative for Nestin. (c) Potential differences in the rate of DNA replication between neurospheres from the ko-IGF-IR embryos and age-matched embryos from nontransgenic littermates (wt-IGF-IR) were evaluated by using a BrdU-based cell proliferation assay. The percentage of cells replicating DNA is significantly lower in neurospheres from the ko-IGF-IR than in wt-IGF-IR neurospheres. Cell-cycle distribution (d) and apoptosis by the TUNEL assay (e) in proliferating neurospheres were evaluated by flow cytometry. The results in panels $\mathbf{c}, \mathbf{d}$ and e represent average values from three independent experiments in triplicates $(n=9)$. Asterisk $\left({ }^{*}\right)$ indicates values that are statistically different from wt-IGF-IR $(P \leqslant 0.05)$

wt-IGF-IR neurospheres were maintained in suspension culture for several weeks, and only a small fraction of cells showed signs of early neuronal differentiation (Figure $2 \mathrm{~b}$ ). Although ko-IGF-IR and wt-IGF-IR progenitors behave in a very similar manner, a detailed evaluation of cell-cycle distribution revealed some differences between the two populations. The percentage of cells replicating DNA (BrdU incorporation) was significantly lower in neurospheres from the ko-IGF-IR embryos (Figure 2c). This statistically significant $\left({ }^{*}\right)$ lower rate of DNA replication was measured in freshly passed secondary neurospheres, which were incubated in growth-supporting medium (GM). The lower level of BrdU incorporation observed in ko-IGF-IR neurospheres was accompanied by a shift in cell-cycle distribution, with an accumulation of cells in $\mathrm{G}_{1}$. Quantitatively, $61.1 \%$ of the wt-IGF-IR progenitors were found in $\mathrm{G}_{1}$, percentage that increased to $70.4 \%$ in ko-IGF-IR progenitors. This $9 \%$ increase in $\mathrm{G}_{1}$ phase accumulation was accompanied by a proportional decrease in cells found in $S$ and $G_{2} / M$ phase (Figure 2d), further supporting a decline of cell proliferation in ko-IGF-IR progenitors. Importantly, in this semioptimal growth-supporting conditions the rate of spontaneous apoptosis detected in ko-IGF-IR and wt-IGF-IR neurospheres was very similar, ranging between 2 and $3 \%$ of TUNEL-positive cells in both cell populations (Figure 2e). The basic GM for neurospheres, although not containing IGF-I, is supplemented with high levels of insulin, which at this concentration stimulates IGF-IR. Therefore, supplementation of the GM with IGF-I affected cell-cycle distribution and cell survival of the wt-IGF-IR neurospheres only minimally (data not shown).

In the next series of experiments, we investigated whether the slower rate of cell proliferation observed in the absence of the IGF-IR could affect the ability of neural progenitors to differentiate in vitro. Following attachment and differentiation of neurospheres (Figure 3a), we performed single (Figure 3, 

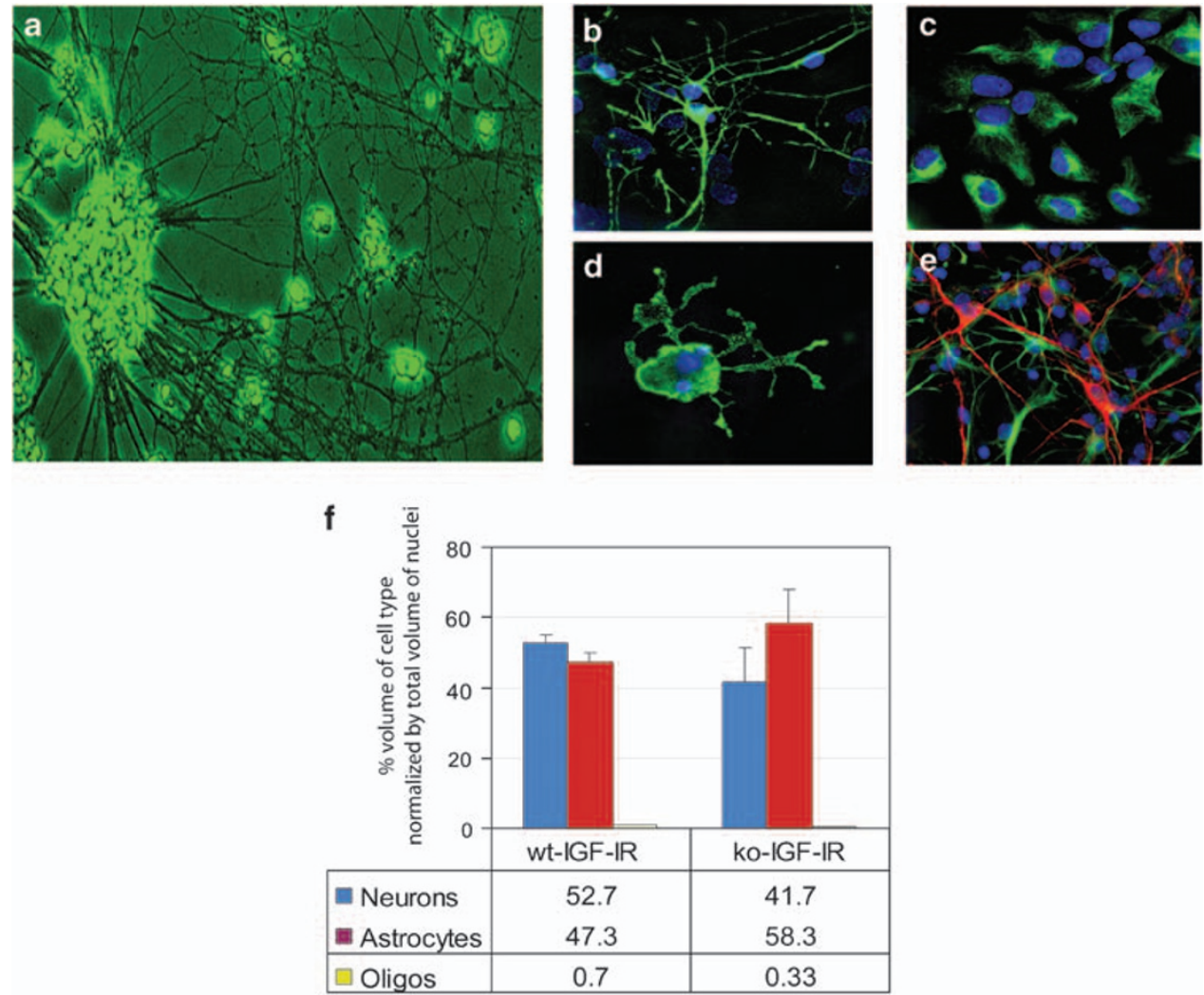

Figure 3 Differentiation of secondary neurospheres. Proliferating neurospheres were induced into differentiation for 5 days. (a) Phase-contrast image illustrating a differentiated neurosphere. Cells were immunolabeled with antibodies for a neuronal marker class III $\beta$-tubulin (b), an astrocytic marker GFAP (c) and an oligodendrocytic marker GalC (galactocerebrosidase) (d). The image depicted in panel e illustrates double labeling of differentiated neurospheres with $\beta$ III-tubulin (rhodamine) and GFAP (fluorescein). In all panels nuclei are counterstained with DAPI (blue). (f) Quantification of the ratios between different cell populations in differentiated neurospheres. The average results from three independent experiments in triplicate $(n=9)$ were collected at day 5 after differentiation of the wt-IGF-IR and ko-IGF-IR neurospheres

panels $b-d$ ) and double (panel e) labeling to detect specific markers for neurons, astrocytes and oligodendrocytes. At 5 days after the induction of differentiation, three morphologically distinct cell populations can be detected. These cells were additionally characterized by immunolabeling with antibodies for class III $\beta$-tubulin (panel b), GFAP (panel c) and GalC (panel d). Double labeling of $\beta$ III-tubulin (rhodamine) and GFAP (fluorescein) showed the abundance of neurons and astrocytes in the neurospheres (panel e). Quantitatively, we observed a slight increase in the astrocyte/neuron ratio, which could be associated with the lack of IGF-IR (Figure 3f). In respect to oligodendrocytes, we have observed a twofold decrease (from 0.7 to $0.33 \% ; n=3$ ) of this cell type in differentiated neurospheres from IGF-IR knockouts.

Growth and survival responses of neural progenitors expressing JCV T-antigen. Once the basic growth, survival and differentiation responses of ko-IGF-IR neural progenitors had been evaluated, we investigated how ectopic expression of JCV T-antigen could affect the fate of cells lacking IGF-IR. The relevance of this question is supported by experiments showing that IGF-IR is required for both JCV T-antigen ${ }^{25}$ and SV40 T-antigen-mediated cellular transformation. ${ }^{14}$ This could also be quite relevant for the development of medulloblastomas, in which JCV T-antigen has been shown to trigger tumor formation in transgenic animals containing the early coding region of JCV under the control of its own promoter. $^{2,26}$ In humans, JCV genomic sequences have been amplified and expression of T-antigen has been detected in clinical samples of medulloblastoma. ${ }^{27,28}$ In addition, medulloblastomas are characterized by a robust upregulation of different components of the IGF-IR signaling pathway, ${ }^{19,25}$ which could enable functional interplay between the IGF-IR and JCV T-antigen in the development of a malignant phenotype. Results depicted in Figure 4a show lower levels of Survivin by western blot in ko-IGF-IR neurospheres compared with wt-IGF-IR. Interestingly, this decrease in Survivin was not affected by ectopic induction of T-antigen expression, which has been previously shown to upregulate Survivin in JCV-infected astrocytes and oligodendrocytes. $^{23}$ In contrast, wt-IGF-IR neurospheres transfected with JCV T-antigen were characterized by a significant threefold upregulation of Survivin in comparison with control neurospheres transfected with empty vector (EV; Figure 4b). In wt-IGF-IR neurospheres, transient expression of JCV T-antigen resulted in activation of cell-cycle progression, which resulted in a decrease of cells in $\mathrm{G}_{1}$ from 64 to $41 \%$ ( $36 \%$ decrease), an increase of cells in $\mathrm{S}$ phase from 21 to $31 \%$ (47\% increase) and an increase of cells in $\mathrm{G}_{2} / \mathrm{M}$ from 14 to $25 \%$ (79\% increase) (Figure $4 \mathrm{c}$ ). 


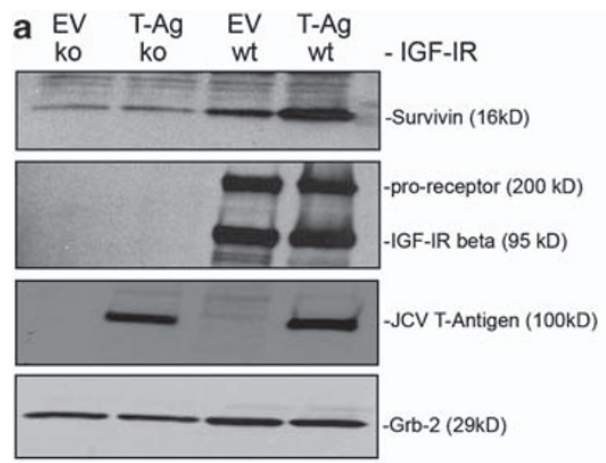

b
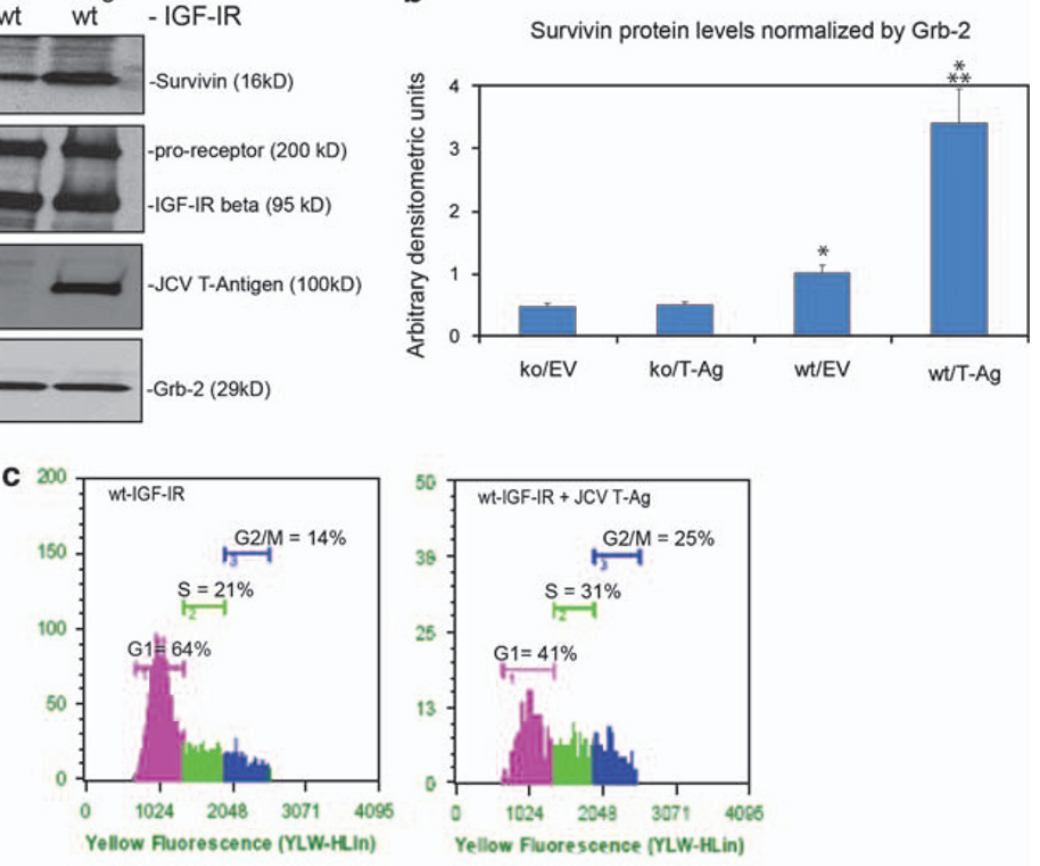

\begin{tabular}{|c|c|c|c|c|c|c|}
\hline & G1 & $\mathbf{s}$ & & & $\%$ T-Antigen expressing cells & Transfection efficiency (GFP) \\
\hline wt-IGF-IR & & 64 & 21 & 14 & 0 & \\
\hline wt-IGF-IR/JCVT & & 41 & 31 & 25 & $57.5+1-4.6$ & $68.3+1-6 \%$ \\
\hline IGF-IR $\%$ & & 71 & 17 & 11 & 0 & \\
\hline IGF-IR-//IJCVT & & - & - & - & $7.1+/-1.3$ & $64.5+1-7 \%$ \\
\hline
\end{tabular}

Figure 4 Effects of JCV T-antigen expression on neural progenitor neurospheres. (a) Western blot analysis showing Survivin levels in wt-IGF-IR and ko-IGF-IR secondary neurospheres in the presence and absence of T-antigen (T-Ag) ectopic expression. (b) Densitometric analysis of western blots depicted in panel a. Data represent average results from three independent experiments $(n=3)$. ${ }^{*}$ indicates values significantly different from ko-IGF-IR neurospheres transiently transfected with a control empty vector (EV). ${ }^{*}$ indicates values significantly different from wt-IGF-IR neurospheres transiently transfected with EV $(P \leqslant 0.05)$. (c) Cell-cycle distribution in wt-IGF-IR neurospheres transiently transfected either with the JCV T-antigen containing plasmid expression vector (pcDNA3zeo/JCV-T-Ag) or with pcDNA3zeo empty vector (EV). Cell-cycle analysis was evaluated by flow cytometry. (d) Comparison of cell-cycle distribution in combination with transfection efficiency (GFP) and the evaluated percentage of cells expressing JCV T-antigen. In the presence of IGF-IR, the expression of T-antigen shifted neural progenitors from $\mathrm{G}_{1}$ toward $\mathrm{S}_{-} \mathrm{G}_{2} \mathrm{M}$ phase of the cell cycle, which increased their rate of cell proliferation. In the absence of the IGF-IR, the low number of JCV T-antigen-positive cells did not allow evaluation of cell-cycle distribution. Note that despite of very similar levels of transfection efficiency between wt-IGF-IR and ko-IGF-IR neurospheres, ko-IGF-IR neurospheres show very low numbers of cells expressing T-antigen. The results represent average values from three independent experiments $(n=3)$

Note that cell-cycle distribution was evaluated only in the population of cells expressing T-antigen. In this experiment, T-antigen-positive cells (green) were gated first, and then the DNA content (propidium iodide; red) was measured in the selected cell population. Interestingly, in this transient transfection experiment, $57.5 \%$ of wt-IGF-IR cells were T-antigen positive (Figure 4d). However, when T-antigen was expressed in ko-IGF-IR neurospheres, the number of T-antigen-positive cells dropped to $7 \%$ (Figure $4 d$ ). This low number of T-antigen-positive cells in ko-IGF-IR neurospheres was not related to the efficiency of transfection because both cell types were nucleofected with a similar efficiency ( $>60 \%$; Figure $4 d$ ).

The next series of experiments was aimed to determine the importance of the IGF-IR in the fate of neurospheres exposed to JCV T-antigen. At $16 \mathrm{~h}$ after the introduction of T-antigen into ko-IGF-IR neurospheres, almost all
T-antigen-positive cells were apoptotic (TUNEL positive). In contrast, T-antigen-expressing cells from the wt-IGF-IR neurospheres were TUNEL negative (Figure 5a). Quantitatively, $96 \%$ of the cells expressing T-antigen were apoptotic in ko-IGF-IR neurospheres, in contrast with only $4 \%$ of apoptotic cells detected in wt-IGF-IR/T-antigen-expressing neurospheres (Figure 5b). Interestingly, the level of apoptosis in IGF-IR knockout neurospheres after reconstitution of Survivin does not quite match the levels seen in wild-type IGF-IR progenitors. This may reflect the presence of another survival pathway, but can also be associated with the efficiency of transfection during delivery of the Survivin expression vector or the efficiency of the siRNA treatment. Importantly, simultaneous expression of T-antigen and Survivin cDNAs in ko-IGF-IR neurospheres decreased T-antigen-induced apoptosis from 96 to $20 \%$. Finally, we performed western blots for Survivin, T-antigen and IGF-IR 

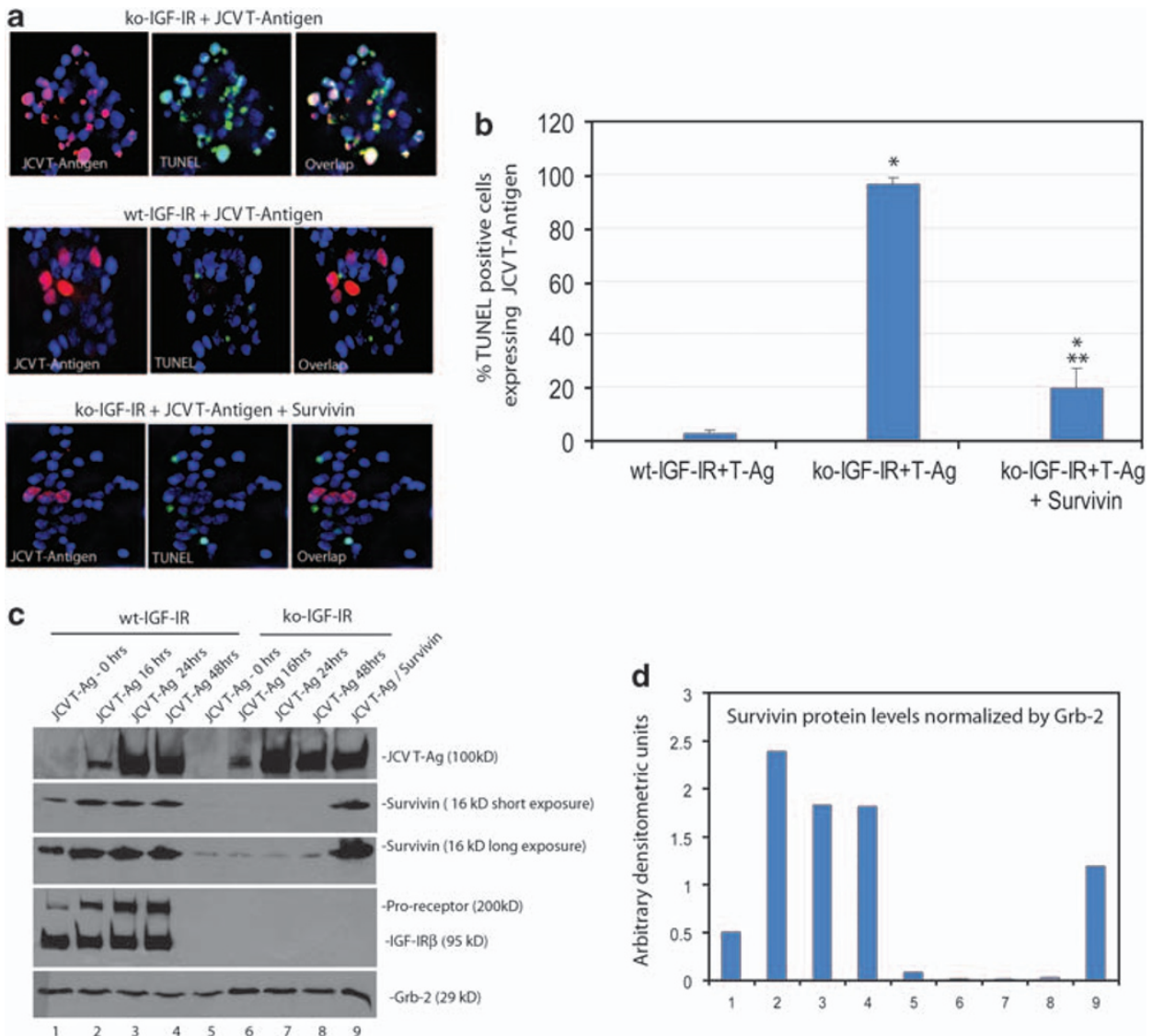

Figure 5 JCV T-antigen-mediated apoptosis in secondary neurospheres. (a) ko-IGF-IR and wt-IGF-IR secondary neurospheres were transfected with JCV T-antigen and/ or Survivin expression vectors. Immunolabeling with anti-T-antigen mouse monoclonal antibody in combination with TUNEL assay detected massive apoptosis in JCV T-antigen-expressing cells from ko-IGF-IR neurospheres $16 \mathrm{~h}$ after transfection. In the presence of transient expression of Survivin, ko-IGF-IR neurospheres are protected from JCV T-antigen-induced apoptosis. (b) Quantitative evaluation of TUNEL-positive cells depicted in panel a. Note that percentage of apoptosis has been determined in the population of cells that was JCV T-antigen positive. Data represent average values from three separate experiments in duplicate $(n=6)$. * indicates values significantly different from wt-IGF-IR/T-Ag neurospheres. ${ }^{* *}$ indicates values significantly different from ko-IGF-IR/T-Ag neurospheres $(P \leqslant 0.05)$. (c) Evaluation of JCV T-antigen, Survivin and IGF-IR protein levels for the experiments depicted in panels $\mathbf{a}$ and $\mathbf{b}$. (d) Densitometric analysis of the Survivin blot depicted in panel $\mathbf{c}$

in (panel c) and quantified their levels (panel d) in the neurospheres used for cell-cycle analysis and detection of apoptosis.

If indeed, IGF-I-dependent activation of Survivin is responsible for the fate of neural progenitors in which JCV T-antigen is expressed, we should be able to sensitize wt-IGF-IR neurospheres to T-antigen by downregulating Survivin expression. This possibility was tested in experiments depicted in Figure 6. First we evaluated the effectiveness of the pool of siRNAs designed against mouse Survivin mRNA. As shown in Figure $6 a$, Survivin levels were downregulated by specific siRNA for Survivin ( $48 \mathrm{~h}$ after the nucleoporation delivery of the siRNA). In the control reaction, irrelevant siRNA designed to target GAPDH mRNA did not affect the levels of Survivin.

Next, we nucleofected Survivin siRNA in combination with a JCV T-antigen expression vector (pcDNA3zeo/JCV-T). Results in Figure $6 \mathrm{~b}$ illustrate that the number of apoptotic cells in wt-IGF-IR neurospheres reached almost $80 \%$, when JCV T-antigen and Survivin siRNA were expressed at the same time. However, when these two constructs were introduced separately, their proapoptotic properties were significantly decreased. JCV T-antigen did not increase the number of apoptotic cells, and siRNA inhibition of Survivin increased apoptosis from the background level (approximately $4 \%$ ) up to $21 \%$. The protein levels for JCV T-antigen and Survivin in this transient transfection experiment are shown in Figure 6b, lower panel. A direct connection between apoptosis induced by JCV T-antigen and Survivin is illustrated in Figure 6c, which shows that the majority of T-antigenexpressing cells undergo apoptosis when Survivin is downregulated by specific siRNA, despite of the presence of IGF-IR. In contrast, control GAPDH siRNA did not trigger apoptosis in JCV T-antigen-expressing neural progenitors. Quantitatively, $62 \%$ of the wt-IGF-IR neural progenitors expressing JCV T-antigen underwent apoptosis in the presence of the Survivin siRNA. However, when the Survivin siRNA was replaced with control GAPDH siRNA, all JCV T-antigen-expressing cells were TUNEL negative (Figure 6c). For comparison, we show another example of the ko-IGF-IR progenitors expressing T-antigen, in which almost all T-antigen-expressing cells are apoptotic. As before, the levels of apoptotic cells in knockout progenitors on siRNA silencing of Survivin does not exactly match the wild type, which may hint the presence of another survival pathway; however, the 

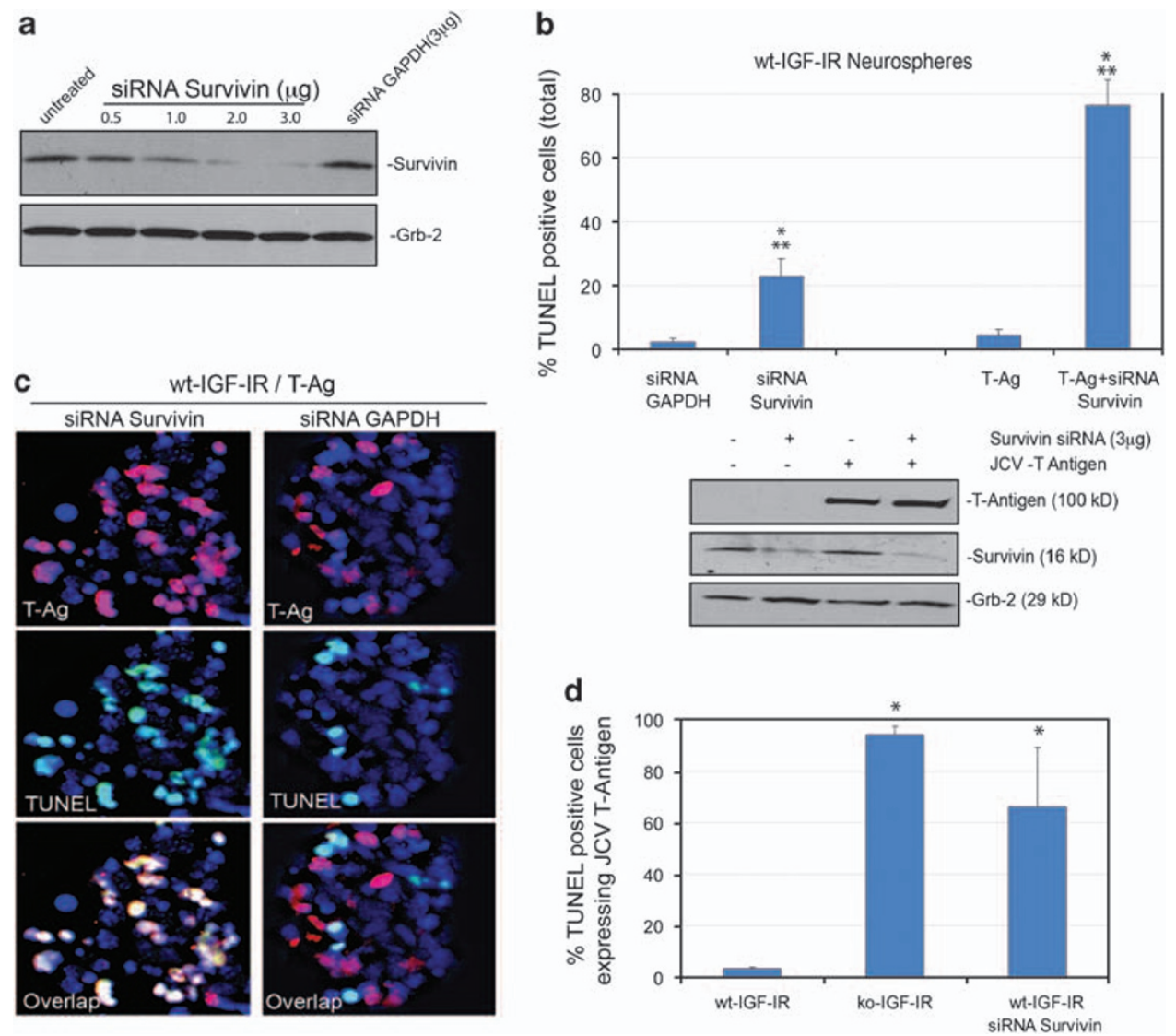

Figure 6 Effects of siRNA downregulation of Survivin on JCV T-antigen-induced apoptosis in secondary neurospheres. (a) Western blot analysis showing a dosedependent siRNA downregulation of Survivin. Untreated and nucleoporated neurospheres with irrelevant GAPDH siRNA were used as controls. The blot re-probed with antiGrb-2 antibody was used as loading control. (b) Effects of nucleoporation-mediated introduction of siRNAs and JCV T-antigen expression vector into wt-IGF-IR neurospheres. Rate of apoptosis after nucleoporation in all cells, independently from the presence or absence of JCV T-antigen, was evaluated by TUNEL assay. Date represent average values from three separate experiments in duplicate $(n=6) .{ }^{*}$ indicates values significantly different from neurospheres nucleofected with GAPDH siRNA. ** indicates values significantly different from neurospheres nucleofected with JCV T-antigen (T-Ag) $(P \leqslant 0.05)$. (b, lower panel) JCV T-antigen and Survivin protein levels for the experiment shown in upper panel. (c) TUNEL and JCV T-antigen double labeling from wt-IGF-IR secondary neurospheres nucleofected with either Survivin or GAPDH siRNAs showed massive apoptosis in wt-IGF-IR neurospheres nucleofected with both JCV T-antigen and Survivin siRNA. In the absence of siRNA to Survivin, JCV T-antigen-expressing wtIGF-IR neurospheres transfected with the irrelevant GAPDH siRNA are TUNEL negative. (d) Quantitative evaluation of TUNEL-positive cells depicted in panel c. Note that apoptosis has been determined in T-antigen-positive cells. Data represent average values from three separate experiments in triplicate $(n=9)$. * indicates values significantly different from wt-IGF-IR/GAPDH siRNA neurospheres $(P \leqslant 0.05)$

study of other IAP members, such as CIAP-1, was negative and the levels of $\mathrm{Bcl}-2$ were not significantly different. A plausible explanation could be the efficiency of siRNA delivery.

Our results indicate that the upregulation of Survivin in response to JCV T-antigen is critical for the survival of cells carrying this viral oncoprotein. Therefore, it is possible that other well-documented cellular responses to JCV T-antigen such as activation of cellular proliferation, immortalization and the induction of genomic instability could be compromised if T-antigen-expressing cells fail to activate Survivin. In the absence of Survivin expression, observed in cells lacking the IGF-IR, expression of JCV T-antigen triggers massive apoptosis of neural progenitors.

mTOR contribution to JCV T-antigen-mediated activation of Survivin. Because the transcriptional activation of Survivin through IGF-I stimulation has been shown to require mTOR signaling in prostate cancer cells, we asked whether this particular signaling branch from the IGF-IR supports survival of JCV T-antigen-expressing neural progenitors. Results in Figure 7a show that endogenous levels of Survivin, in IGF-IR expressing control progenitors (EV), are strongly downregulated by $10 \mu \mathrm{M}$ mTOR inhibitor, rapamycin, which at this concentration blocked completely mTORdependent phosphorylation of p70Sp6 kinase. In neurosphere cultures, transiently expressing JCV T-antigen levels of Survivin were at list twofold higher than in control cultures $(E V)$, and the rapamycin treatment also downregulated Survivin protein levels in JCV T-antigen-expressing cells (T-antigen + Rap). Note however that in the presence of JCV $T$-antigen the remaining levels of Survivin were still higher than the levels detected in the control untreated neurosphere cultures (Figure 7a, compare lanes T-Ag + Rap and EV). Next, we have evaluated how the rapamycin treatment affected survival of neural progenitors in the presence and 

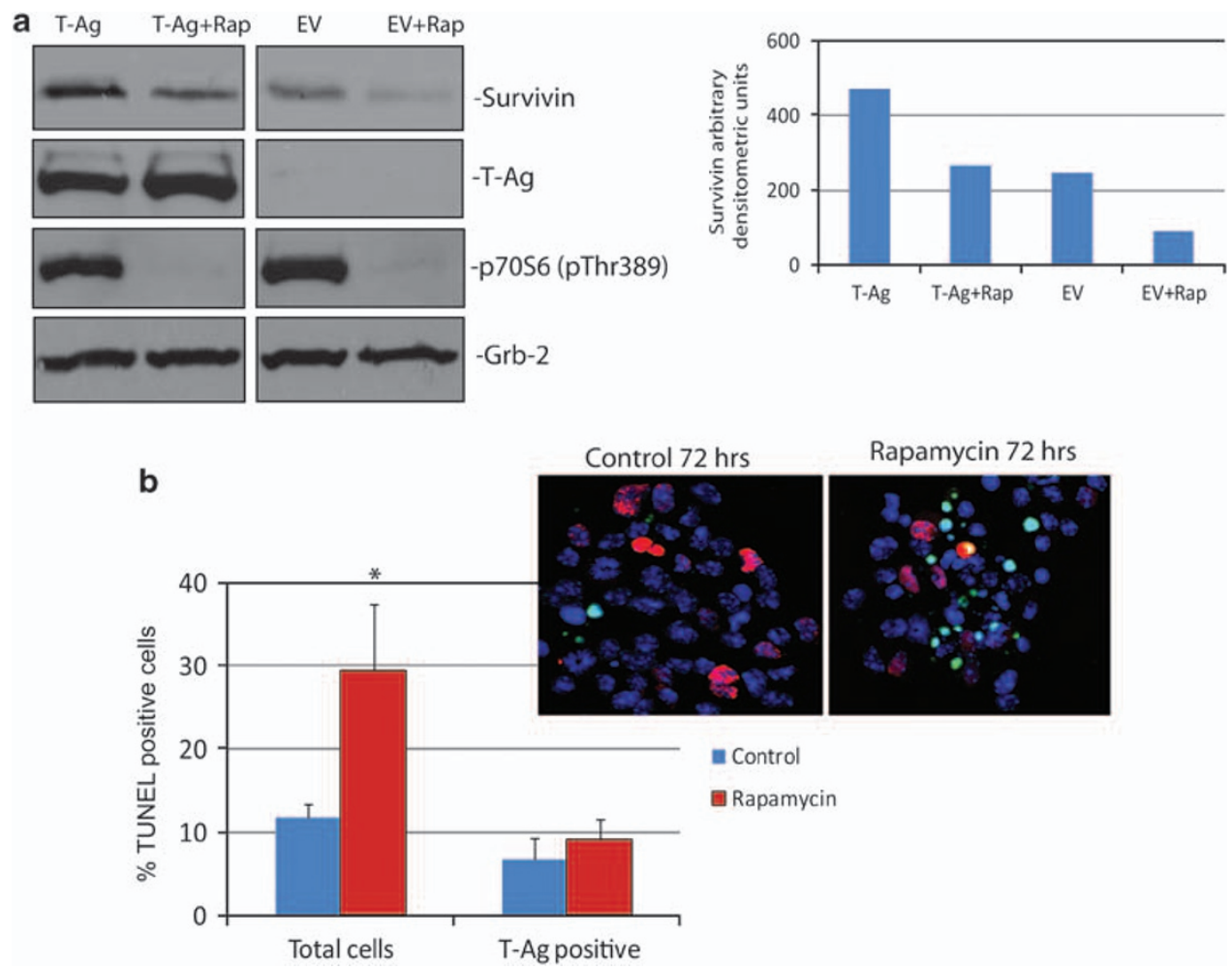

Figure 7 Evaluation of mTOR contribution to JCV T-antigen-mediated activation of Survivin. (a) Western blot analysis based on protein lysates isolated from the wt-IGF-IR neurospheres. The cells were transfected with either T-antigen-containing vector (pcDNA3zeo/JCV-T) or control empty vector (EV; pcDNA3zeo) and were cultured in the presence or absence of $10 \mu \mathrm{M}$ rapamycin for $72 \mathrm{~h}$. The resulting protein blots were probed with anti-Survivin, anti-T-antigen and anti-p70Sp6 kinase antibodies. Anti-Grb-2 antibody was used to monitor loading conditions. The histogram below represents densitometric analysis of the Survivin blot normalized by the level of Grb-2. (b) Immunocytofluorescent evaluation of wt-IGF-IR neurospheres transiently expressing T-antigen. The cells were simultaneously labeled for apoptosis (TUNEL assay, green fluorescence) and for T-antigen (rhodamine). Nuclei are counterstained with DAPI. The histogram shows quantitative evaluation of TUNEL-positive cells depicted and was performed for T-antigen-negative and T-antigen-positive cells separately. Data represent average values from two experiments in triplicate $(n=6)$. ${ }^{*}$ indicates values significantly different from controls $(P \leqslant 0.05)$

absence of JCV T-antigen. Results depicted in Figure $7 \mathrm{~b}$ show the detection of TUNEL-positive cells at $72 \mathrm{~h}$ after transient delivery of the JCV T-antigen expression vector. As illustrated by the inset to Figure $7 \mathrm{~b}$, in the absence of rapamycin, most of JCV T-antigen-negative (blue) and T-antigen-positive (red) cells were TUNEL negative. Quantitatively, $11 \%$ of T-antigen-negative cells and $7 \%$ of T-antigen-positive cells were undergoing apoptosis in control conditions. After rapamycin treatment, the percentage of TUNEL-positive cells increased from 11 to $30 \%$ among T-antigen-negative population, and from 7 to $9 \%$ among the population of T-antigen-expressing cells. These data suggest that although the mTOR inhibition downregulates Survivin protein levels in T-antigen-expressing neural progenitors, the remaining level of this antiapoptotic protein seems to be high enough to sustain cell survival in the presence of T-antigen.

\section{Discussion}

Medulloblastomas constitute the most frequent intracranial tumor of childhood. These highly malignant tumors originate from neuronal precursors in the external granule layer of the developing cerebellum or in the periventricular germinal matrix and velum medullare. ${ }^{29}$ Although inactivating mutations of the Patched gene, which encodes the Sonic hedgehog receptor, have been found in about $10 \%$ of sporadic medulloblastomas, ${ }^{30}$ other mechanisms have been considered as potential contributing factors in the development of medulloblastomas, including JCV, ${ }^{18,27,31}$ and high levels of expression of different components of the IGF-IR signaling system. ${ }^{19}$ In fact, a substantial body of evidence points to the function of polyomaviruses in human carcinogenesis. ${ }^{2,32}$ All three polyomaviruses (BKV, SV40, and JCV) express large T-antigen, have the ability to transform cells in vitro, are tumorigenic in experimental animals and have been found in a variety of human cancers including medulloblastoma. In addition, epidemiological studies show that up to $90 \%$ of the human population is seropositive for $\mathrm{JCV},{ }^{33}$ raising the possibility that this neurotropic virus may be a common factor in tumor formation worldwide.

Because it is still not clear why polyomavirus T-antigens require IGF-IR in the process of cellular transformation, we have investigated this phenomenon in neural progenitors from IGF-IR knockout mouse embryos (ko-IGF-IR) and from wildtype nontransgenic littermates (wt-IGF-IR). We have found that the brain and DRG from ko-IGF-IR embryos were characterized by very low levels of the antiapoptotic protein, Survivin, accompanied by elevated numbers of apoptotic neurons. In addition, JCV T-antigen expressed in wt-IGF-IR neural progenitors tripled the expression of Survivin, 
accelerated cell proliferation and improved cell survival. Interestingly, these effects were mediated only partially by the mTOR pathway because treatment with rapamycin did not decrease the levels of Survivin effectively enough to induce apoptosis in T-antigen-expressing cells. Importantly, JCV T-antigen failed to induce elevated Survivin expression in ko-IGF-IR progenitors and its expression triggered massive apoptosis. Ectopic expression of Survivin cDNA efficiently protected ko-IGF-IR progenitors from T-antigen-induced apoptosis, and finally, downregulation of Survivin by a specific siRNA activated apoptosis in wt-IGF-IR progenitors expressing T-antigen. Therefore, reactivation of Survivin by JCV T-antigen may represent a basic cellular response to the expression of T-antigen without which other cellular effects, including those associated with immortality and transformation, could be compromised.

We should not dismiss, however, the high redundancy found in biological systems, among which cancer cells represent probably the most spectacular example of accelerated selection and cellular adaptation to new environmental, genetic and metabolic challenges. It has been shown for instance that one of the late-passage clones of MEFs with targeted disruption of the IGF-IR gene ( $R^{-}$cells), stably transfected with SV40 T-antigen, underwent malignant transformation despite the absence of the IGF-IR. ${ }^{16}$ Comparison between mRNA microarray libraries from early and late passages of this particular clone showed, among other aberrations, exceptionally strong expression of ErbB-3. Interestingly, ErbB-3, which is a family member of membrane-associated tyrosine kinase receptors (ErbB1-4), has been shown to promote cell survival and tumor formation. ${ }^{16,34}$ Of high relevance to our study, constitutively active ErbB-2/ ErbB-3 heterodimers found in breast cancer cells showed a strong ability of activating expression of Survivin, which in turn rendered drug resistance in these cells. ${ }^{16}$ Therefore, one could speculate that the requirement for IGF-IR in T-antigenmediated transformation of neuronal precursors may not be absolute, and could be replaced for instance by ErbB-3 overexpression. Incidentally, two other members of ErbB family, ErbB-2 and ErbB-4, have been found in over $70 \%$ of medulloblastoma cases, and their presence correlated with poor prognosis. ${ }^{35}$

Another effect of T-antigen on the IGF-IR signaling system that could contribute to cellular transformation is its interaction with IRS-1. In addition to its known metabolic and growthpromoting functions, IRS-1 is suspected to have a function in malignant transformation, presumably by amplifying the IGFIR signal. The first convincing evidence indicating transforming potential of IRS-1 was showed again in $\mathrm{R}^{-}$cells, which are resistant to transformation by T-antigen. However they acquire a transformed phenotype following co-transfection with IRS-1 and T-antigen. ${ }^{14,36}$ In a similar manner, T-antigen from JCV also required the presence of IGF-IR-IRS-1 signaling axis for transformation. ${ }^{17}$ Interestingly, we have found nuclear IRS-1 in cells expressing JCV T-antigen ${ }^{7}$ and in JCV T-antigen-positive medulloblastoma clinical samples. ${ }^{17,25}$ The biological relevance of nuclear IRS-1 has been studied extensively during the last several years. In fibroblasts stimulated with IGF-I, nuclear IRS-1 has been found in association with upstream binding factor 1 (UBF1, regulator of
RNA polymerase I), which coincided with increased rRNA synthesis. ${ }^{37}$ In addition, a direct connection between IRS-1 and homologous recombination-dependent DNA repair (HRR) has recently been proposed. ${ }^{9}$ This new signaling interplay involves the perinuclear binding between hypophosphorylated IRS-1 and the major enzymatic component of HRR, Rad51. IRS-1 tyrosine phosphorylation attenuated IRS-1 binding to Rad51 allowing efficient translocation of Rad51 to the sites of damaged DNA, which lead to the increased contribution of HRR in DNA repair of double-strand breaks. In JCV T-antigen-positive cells, however, IRS-1 translocates to the nucleus, where it has been found in complex with Rad51 at sites of damaged DNA preventing faithful DNA repair. The presence of nuclear IRS-1/Rad51 complexes was strongly associated with the inhibition of HRR and with the increased incidence of spontaneous mutations in cells expressing JCV T-antigen. ${ }^{10}$ Therefore, it is reasonable to speculate that because of the presence of T-antigen/IGF-I/ Survivin signaling axis, T-antigen-expressing cells can survive replicative DNA damage, unfaithful DNA repair and accumulation of spontaneous mutations.

Another pathological aspect of Survivin activation by JCV T-antigen has been raised recently. In addition to cellular transformation, reactivation of Survivin by JCV T-antigen can be a critical step in prolonging cell survival, which allows JCV to complete its replication cycle. Such a strong reactivation of the normally dormant Survivin has been observed in AIDS-related PML cases and in primary oligodendrocyte and astrocyte cultures infected in vitro, and expressing JCV T-antigen. ${ }^{23}$ Figure 8 shows a schematic representation of the results from this work and other hypothetical scenarios.

In summary the results from this study show an important function for the IGF-IR in the process of Survivin reactivation by JCV T-antigen, which may represent a critical step in the transformation and proliferation of neural progenitors in vitro and in vivo. Unraveling the interactions and regulation between these proteins may be a critical step in understanding the pathogenesis of medulloblastomas and may provide a potential target for the treatment of these devastating neoplasms.

\section{Materials and Methods}

Knockout IGF-IR transgenic mice and PCR genotyping. Transgenic mice heterozygotes for targeted disruption of the IGF-IR gene have been kindly provided by Dr. Efstradiadis, Columbia University, NY, USA. Mouse embryos (embryonic day 16) were obtained as a result of the breeding between IGF-IR knockout (ko) heterozygotes $( \pm$ ). In the same litter, ko-IGF-IR mice are about onethird of the size of nontransgenic littermates. ko-IGF-IR embryos die shortly after birth as a result of respiratory failure; otherwise the dwarf embryos look normal. Heterozygotes do not have phenotype and are fully fertile. ${ }^{38} \mathrm{~A}$ standard PCR has been routinely performed to identify IGF-IR knockout heterozygotes for further breeding purposes. A small piece of tail was cut from 3-week-old mice and was used for the extraction of genomic DNA using the protocol described in our previous work. ${ }^{39}$ To detect neomycin knockout transgene, we have used the following primers: IGF-IR neo forward, 5'-CAGGACATAGCGTTGGCTACCC-3'; IGF-IR neo reverse, 5' -GGA CCTTCTACAAGGTGGGGAC-3' (Integrated DNA Technologies, San Diego, CA, USA).

Immunohistochemistry of IGF-IR knockout and wild-type embryos. Freshly isolated embryos were fixed in formalin and embedded in paraffin. Sections (4- $\mu \mathrm{m}$ thick) were cut, placed in electromagnetically charged slides and stained with $\mathrm{H} \& \mathrm{E}$ for histological evaluation. Immunohistochemistry was performed using the avidin-biotin-peroxidase complex system according to the manufacturer's 


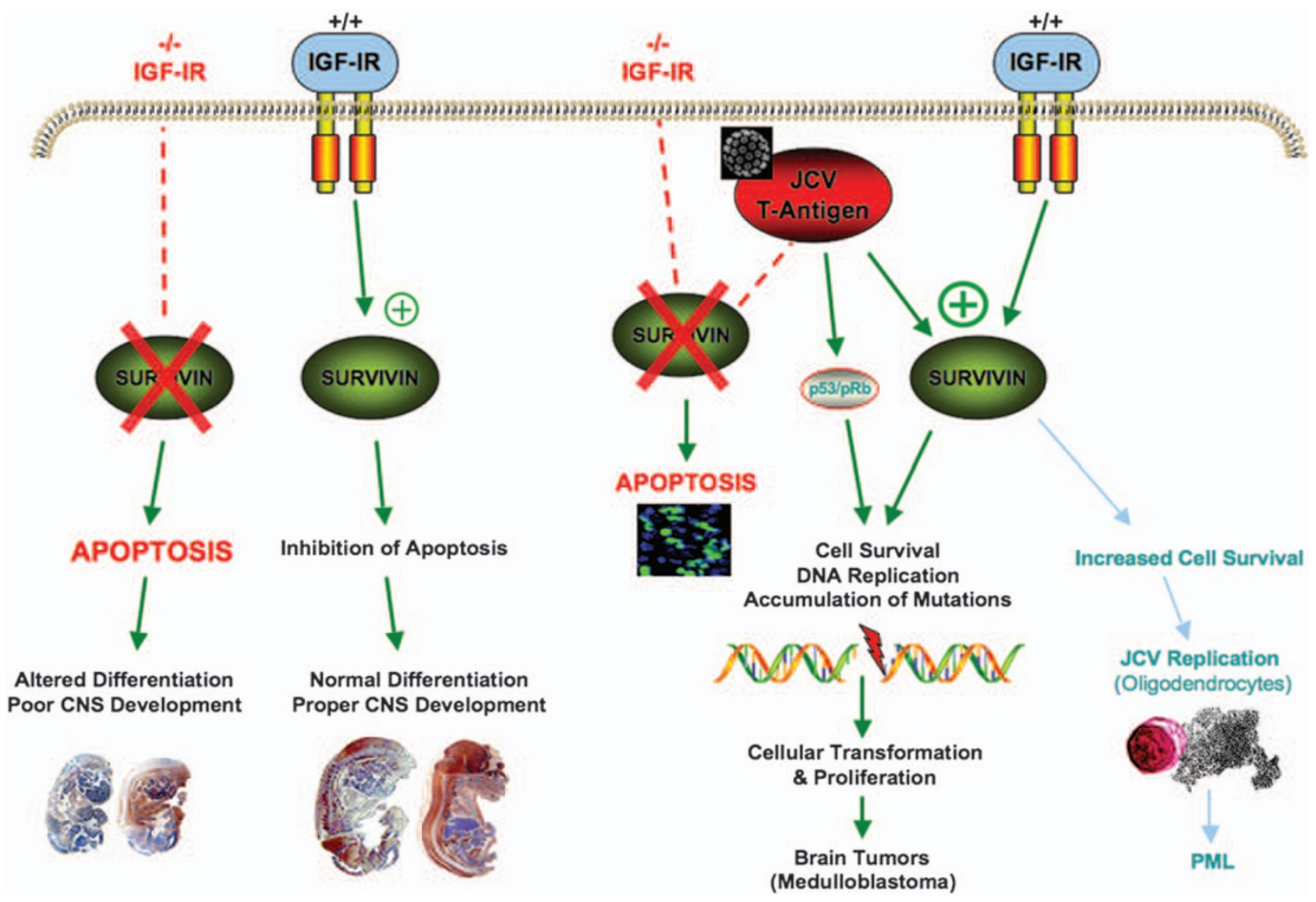

Figure 8 Schematic representation of IGF-IR/T-antigen/Survivin interactions. IGF-IR knockout embryos show low levels of the antiapoptotic protein Survivin, which results in abundant apoptosis and small and poorly differentiated CNS. In contrast wild-type animals with intact receptor show abundant Survivin and normal CNS growth and differentiation. In IGF-IR knockout progenitors lacking Survivin, the introduction of JCV T-antigen results in massive apoptosis. However in the presence of IGF-IR and high levels of Survivin, T-antigen expression results in prolonged cell survival, which results in proliferation, eventually leading to transformation and possibly to the development of medulloblastomas. In another hypothetical scenario, under immunosuppressive conditions, the prevention of apoptosis and its resulting prolonged cellular survival will allow time for JCV active viral replication, leading to the development of PML

instructions (Vectastain Elite ABC kit; Vector Laboratories Inc., Burlingame, CA, USA). Our modified protocol included deparaffinization in xylenes, rehydration through descending grades of alcohol up to water and nonenzymatic antigen retrieval in $0.01 \mathrm{~mol} / /$ sodium citrate buffer $\left(\mathrm{pH} \mathrm{6.0)}\right.$ ) heated to $95^{\circ} \mathrm{C}$ for $40 \mathrm{~min}$ in a vacuum oven. After a cooling period of $30 \mathrm{~min}$, the slides were rinsed in phosphatebuffered saline (PBS) and treated with $3 \% \mathrm{H}_{2} \mathrm{O}_{2}$ in methanol for 25 min to quench endogenous peroxidase. Sections were then rinsed with PBS and blocked with $5 \%$ normal horse serum (for mouse monoclonal antibodies) or goat serum (for rabbit polyclonal antibodies) in $0.1 \% \mathrm{PBS} /$ bovine serum albumin (BSA) for $2 \mathrm{~h}$ at room temperature. Primary antibodies were incubated overnight at room temperature in a humidifier chamber. Primary antibodies used in this study included mouse monoclonal antibodies for Survivin (Clone D-8, 1: 100 dilution; Santa Cruz Biotechnology, Santa Cruz, CA, USA), BCl-2 (Clone C-2, 1:100 dilution; Santa Cruz Biotechnology), GFAP (Clone 62F, 1: 100 dilution; DAKO, Carpinteria, CA, USA) and class III $\beta$-tubulin (Clone TuJ1, 1:500 dilution; Covance, Berkeley, CA, USA) and rabbit polyclonal antibodies for cIAP-1 (ab25939, 1:500 dilution; Abcam, Cambridge, MA, USA) and Nestin (PRB-315C, 1:500 dilution; Covance). Biotinylated secondary anti-mouse or anti-rabbit antibodies were incubated for $1 \mathrm{~h}$ at room temperature $(1: 200$ dilution). Finally, sections were incubated with avidin-biotin complexes (ABC Elite kit; Vector Laboratories) for $1 \mathrm{~h}$ at room temperature, rinsed with PBS and developed with diaminobenzidine (DAB Tablets; Sigma, St. Louis, MO, USA). Finally, the sections were counterstained with hematoxylin and mounted with Permount (Fisher Scientific, Fair Lawn, NJ, USA).

TUNEL assay. Apoptotic cells were identified by TUNEL assay, by using the ApopTag methodology according to the manufacturer's instructions (ApopTag
Peroxidase In Situ Detection Kit; Millipore, Billerica, MA, USA). Briefly, sections were deparaffinized, rehydrated and endogenous peroxidase quenched as described for immunohistochemistry. The sections were then pretreated with Proteinase $\mathrm{K}$ for $15 \mathrm{~min}$ at room temperature and treated with an equilibration buffer and TdT enzyme for $10 \mathrm{~s}$ and $1 \mathrm{~h}$, respectively. Finally sections were incubated with an anti-digoxigenin conjugate, washed with PBS and developed with diaminobenzidine (Sigma), counterstained with hematoxylin and mounted with Permount. For the quantification of apoptosis in the brain, all positive cells were manually counted in 20 fields of $\times 400$ magnification and divided by the total number of cells in the same fields for the percentage of positivity (labeling index). The same methodology was applied to determine the labeling index of DRG, with the difference that all cells in 8 DRG were counted at $\times 40$ magnification instead of 20 fields.

Isolation of neural progenitors and neurosphere cell culture. Using standard technique, ${ }^{40}$ we isolated neural progenitors from the forebrains of 16 -day-old ko-IGF-IR embryos and from nontransgenic littermates. Dissociated by gentle trypsinization single neural cells were plated on nonadherent Petri dishes at densities ranging from $1 \times 10^{2}$ to $1 \times 10^{5}$ cells per $\mathrm{cm}^{2}$. The culture medium used to support proliferation of neural progenitors and the formation of primary neurospheres consisted of NeuroBasal Medium (Gibco-Invitrogen, Carlsbad, CA, USA), B27 supplement (Gibco), N2 supplement (Gibco), Heparin (2 $\mathrm{ng} / \mathrm{ml})$, EGF (20 ng/ml; Invitrogen), bFGF (20 ng/ml; Invitrogen) and Glutamax (Gibco). After 5 days of the continuous growth, primary neurospheres were dissociated to single-cell suspension and plated at low density on nonadherent Petri dishes in the same medium. The resulting secondary neurospheres were used for cell survival and cell proliferation assays, analysis of cell-cycle distribution, protein extraction and immunocytofluorescent labeling. 
Alternatively, secondary neurospheres were plated on poly D-lysine/Laminin-coated glass chambers slides and allowed to differentiate in the NeuroBasal medium containing N2 supplement, Glutamax, forskolin (5 $\mu \mathrm{M}$; Sigma), retinoic acid $(1 \mu \mathrm{M}$; Sigma) and $1 \%$ FBS. The differentiation process was characterized by limited cell death and cell adhesion to the substrate, which occurred during the first several hours. This was followed by cell adhesion, cell spread and the formation of cellular processes. Differentiated neurospheres ( 6 days old) were considered terminally differentiated and have been analyzed by immunofluorescent labeling to discriminate the content of neural cell types.

\section{Immunocytofluorescence and quantification of cellular content.} Proliferating neurospheres were span down on poly-D-lysine/Laminin-coated glass chamber slides by Cytospin 3 (Thermo Shandon, Waltham, MA, USA). Differentiated neurospheres were cultured on poly-D-lysine chamber slides (Nalge Nunc International, Rochester, NY, USA). Both cell types were fixed and permeabilized with the buffer containing $0.02 \%$ Triton X-100 and $4 \%$ formaldehyde in PBS. Fixed cells were washed $3 \times$ in PBS and blocked in $1 \%$ BSA for $30 \mathrm{~min}$ at $37^{\circ} \mathrm{C}$. Neural progenitors, neurons, astrocytes and oligodendrocytes were detected with anti-Nestin (Covance), anti-class III $\beta$-tubulin (Covance), anti-GFAP (Chemicon, Temecula, CA, USA) and anti-GalC (Chemicon) antibodies, respectively. The images were visualized with an inverted Nikon Eclipse TE300 microscope equipped with a Retiga 1300 camera, motorized $z$ axis, and deconvolution software (SlideBook 4, Intelligent Imaging Innovations, Denver, CO, USA). Three-dimensional images of each individual picture were deconvoluted to one two-dimensional picture and resolved by adjusting the signal cutoff to near maximal intensity to increase resolution. Quantification of the volume ratio between different cell populations in differentiated neurospheres was performed using SlideBook 4 software according to the manufacturer's instructions (Intelligent Imaging Innovations). Briefly, series of 11 fluorescent images ( $2 \mu \mathrm{m}$ each) were collected from and the resulting images were processed with the MASK operation software according to manufacturer's recommendations (SlideBook 4, Intelligent Imaging Innovations). First, all red voxels (3D pixels) from the collected 11 plains of the $\beta$ III-tubulin fluorescent image were 'covered' with the blue MASK (3D ruler). Next, all green voxels corresponding either to GFAP or GalC immunolabeling were covered with the blue MASK as well. Finally, the same procedure was applied to DAPI labeled nuclei. To calculate the percentage volume of neurons, astrocytes and oligodendrocytes within any given neurosphere, we normalized the number of $\beta$ IIItubulin voxels (red), GFAP voxels (green) or GalC voxels (green) by the DAPI voxels, and presented the data as an average percentage volume of neurons, astrocytes and oligodendrocytes.

Western blotting. Cultures of secondary neurospheres were lysed for $5 \mathrm{~min}$ on ice with $400 \mu$ of lysis buffer A (50 mM HEPES (pH 7.5), $150 \mathrm{mM} \mathrm{NaCl}, 1.5 \mathrm{mM}$ $\mathrm{MgCl}_{2}, 1 \mathrm{mM}$ EGTA, $10 \%$ glycerol, $1 \%$ Triton X-100, $1 \mu \mathrm{M}$ phenylmethylsulfonyl fluoride, $0.2 \mathrm{mM} \mathrm{Na}$-orthovanadate and $10 \mu \mathrm{g} / \mathrm{ml}$ aprotinin). Total protein extracts $(50 \mu \mathrm{g})$ were separated on a 4-15\% gradient SDS-PAGE (Bio-Rad, Hercules, CA, USA) and transferred to nitrocellulose filters. The following primary antibodies were used: anti-IGF-IR $\beta$ rabbit polyclonal (Santa Cruz Biotechnology), anti-Survivin (Santa Cruz Biotechnology) and a mouse monoclonal anti-SV40 T-antigen, which cross-reacts with JCV T-antigen (Calbiochem, San Diego, CA, USA). Anti-Grb-2 antibody (BD Transduction Laboratories, Franklin Lakes, NJ, USA) was used as a loading marker.

Cell-cycle distribution, DNA replication and apoptosis. All these parameters were evaluated by flow cytometry. Aliquots of cells, $1 \times 10^{6}$ cells per ml, were fixed in $70 \%$ ethanol at $4{ }^{\circ} \mathrm{C}$ for $30 \mathrm{~min}$. The cells were centrifuged at 1600 r.p.m. and the resulting pellets suspended in $1 \mathrm{ml}$ of freshly prepared propidium iodide/RNaseA solution. Cell-cycle distribution was evaluated using Guava EasyCyte flow cytometer and CytoSoft version 4.1 software (Guava Technologies, Hayward, CA, USA). In some experiments, DNA replication was evaluated by BrdU pulse labeling ( $3 \mathrm{~h}$ ) or accumulative labeling ( $24 \mathrm{~h})$, using the ' $/ \mathrm{n}$ Situ Cell Proliferation Kit, FLUOS' (Roche, Molecular Biochemicals, Indianapolis, IN, USA). The percentage of cells replicating DNA was calculated with Guava EasyCyte. TUNEL and Annexin $V$ assays were used as independent methods to evaluate apoptosis in the experimental protocols described above. Both assays have been modified for the use in Guava EasyCyte flow cytometer according to the manufacturer's recommendations with some modifications. ${ }^{23}$
Acknowledgements. We thank past and present members of the Department of Neuroscience and the Center for Neurovirology for their valuable and insightful comments and suggestions. This work was made possible thanks to NIH Grants awarded to KR (R01 CA095518) and LDV (R01 NS055644).

1. Del Valle L, Piña-Oviedo S. HIV disorders of the brain: pathology and pathogenesis. Front Biosci 2006; 11: 718-732.

2. Del Valle L, Gordon J, Ferrante P, Khalili K. JC virus in experimental and clinical brain tumors. In: Khalili K, Stoner GL (eds). Human Polyoviruses Molecular and Clinical Perspectives. First edn Wiley-Liss, Inc.: New York, 2001. pp 409-430.

3. Pipas JM. Common and unique features of T-antigens encoded by the polyomavirus group. J Virol 1992; 66: 3979-3985.

4. Sullivan CS, Tremblay JD, Fewell SW, Lewis JA, Brodsky JL, Pipas JM. Species-specific elements in the large T-antigen $\mathrm{J}$ domain are required for cellular transformation and DNA replication by simian virus 40. Mol Cell Biol 2000; 20: 5749-5757.

5. Saenz-Robles MT, Sullivan CS, Pipas JM. Transforming functions of Simian Virus 40. Oncogene 2001; 20: 7899-7907.

6. Stubdal H, Zalvide J, DeCaprio JA. Simian virus 40 large T-antigen alters the phosphorylation state of the RB-related proteins p130 and p107. J Virol 1996; 70: 2781-2788.

7. Lassak A, Del Valle L, Peruzzi F, Wang JY, Enam S, Croul S et al. Insulin receptor substrate 1 translocation to the nucleus by the human JC virus T-antigen. J Biol Chem 2002; 277: 17231-17238.

8. Reich NC, Levine AJ. Specific interaction of the SV40 T-antigen-cellular p53 protein complex with SV40 DNA. Virology 1982; 117: 286-290.

9. Trojanek J, Ho T, Del Valle L, Nowicki M, Wang JY, Lassak A et al. Role of the insulin-like growth factor I/insulin receptor substrate 1 axis in Rad51 trafficking and DNA repair by homologous recombination. Mol Cell Biol 2003; 23: 7510-7524.

10. Trojanek J, Croul S, Ho T, Wang JY, Darbinyan A, Nowicki M et al. T-antigen of the human polyomavirus JC attenuates faithful DNA repair by forcing nuclear interaction between IRS-1 and Rad51. J Cell Physiol 2006; 206: 35-46.

11. Tan TH, Wallis J, Levine AJ. Identification of the p53 protein domain involved in formation of the simian virus 40 large T-antigen-p53 protein complex. J Virol 1986; 59: 574-583.

12. White MK, Khalili K. Interaction of retinoblastoma protein family members with large T-antigen of primate polyomaviruses. Oncogene 2006; 25: 5286-5293.

13. Porcu P, Ferber A, Pietrzkowski Z, Roberts CT, Adamo M, LeRoith D et al. The growthstimulatory effect of simian virus $40 \mathrm{~T}$-antigen requires the interaction of insulinlike growth factor 1 with its receptor. Mol Cell Biol 1992; 12: 5069-5077.

14. Sell C, Rubini M, Rubin R, Liu JP, Efstratiadis A, Baserga R. Simian virus 40 large tumor antigen is unable to transform mouse embryonic fibroblasts lacking type 1 insulin-like growth factor receptor. Proc Natl Acad Sci USA 1993; 90: 11217-11221.

15. DeAngelis T, Chen J, Wu A, Prisco M, Baserga R. Transformation by the simian virus 40 T-antigen is regulated by IGF-I receptor and IRS-1 signaling. Oncogene 2006; 25 : 32-42.

16. Spence SL, Shaffer AL, Staudt LM, Amde S, Manney S, Terry C et al. Transformation of late passage insulin-like growth factor-I receptor null mouse embryo fibroblasts by SV40 T-antigen. Cancer Res 2006; 66: 4233-4239.

17. Del Valle L, Wang JY, Lassak A, Peruzzi F, Croul S, Khalili K et al. Insulin-like growth factor I receptor signaling system in JC virus T-antigen-induced primitive neuroectodermal tumors - medulloblastomas. J Neurovirol 2002; 8 (Suppl 2): 138-147.

18. Reiss K. Insulin-like growth factor-I receptor - a potential therapeutic target in medulloblastomas. Expert Opin Ther Targets 2002; 6: 539-544.

19. Wang JY, Del Valle L, Gordon J, Rubini M, Romano G, Croul S et al. Activation of the IGF-IR system contributes to malignant growth of human and mouse medulloblastomas. Oncogene 2001; 20: 3857-3868.

20. Urbanska K, Trojanek J, Del Valle L, Eldeen MB, Hofmann F, Garcia-Echeverria C et al. Inhibition of IGF-I receptor in anchorage-independence attenuates GSK-3beta constitutive phosphorylation and compromises growth and survival of medulloblastoma cell lines. Oncogene 2007; 26: 2308-2317.

21. Ambrosini G, Adida C, Altieri DC. A novel anti-apoptosis gene, Survivin, expressed in cancer and lymphoma. Nat Med 1997; 3: 917-921.

22. Vaira V, Lee CW, Goel HL, Bosari S, Languino LR, Altieri DC. Regulation of Survivin expression by IGF-1/mTOR signaling. Oncogene 2007; 26: 2678-2684.

23. Piña-Oviedo S, Urbanska K, Radhakrishnan S, Sweet T, Reiss K, Khalili K et al. Effects of JC virus infection on anti-apoptotic protein Survivin in progressive multifocal leukoencephalopathy. Am J Pathol 2007; 170: 1291-1304.

24. Liu JP, Baker J, Perkins AS, Robertson EJ, Efstratiadis A. Mice carrying null mutations of the genes encoding insulin-like growth factor I (Igf-1) and type 1 IGF receptor (Igf1r). Cell 1993; 75: 59-72.

25. Del Valle L, Enam S, Lassak A, Wang JY, Croul S, Khalili K et al. Insulin-like growth factor I receptor activity in human medulloblastomas. Clin Cancer Res 2002; 8: 1822-1830.

26. Krynska B, Gordon J, Otte J, Franks R, Knobler R, DeLuca A et al. Role of cell cycle regulators in tumor formation in transgenic mice expressing the human neurotropic virus, JCV, early protein. J Cell Biochem 1997; 67: 223-230. 
27. Khalili K, Krynska B, Del Valle L, Katsetos CD, Croul S. Medulloblastomas and the human neurotropic polyomavirus JC virus. Lancet 1999; 353: 1152-1153.

28. Krynska B, Del Valle L, Croul S, Gordon J, Katsetos CD, Carbone M et al. Detection of human neurotropic JC virus DNA sequence and expression of the viral oncogenic protein in pediatric medulloblastomas. Proc Natl Acad Sci USA 1999; 96: 11519-11524.

29. Katsetos CD, Burger PC. Medulloblastoma. Semin Diagn Pathol 1994; 11: 85-97.

30. Rao G, Pedone CA, Del Valle L, Reiss K, Holland EC, Fults DW. Sonic hedgehog and insulin-like growth factor signaling synergize to induce medulloblastoma formation from nestin-expressing neural progenitors in mice. Oncogene 2004; 23 : 6156-6162.

31. Del Valle L, Gordon J, Enam S, Delbue S, Croul S, Abraham S et al. Expression of human neurotropic polyomavirus JCV late gene product agnoprotein in human medulloblastoma. J Natl Cancer Inst 2002; 94: 267-273.

32. Del Valle L, Gordon J, Assimakopoulou M, Enam S, Geddes JF, Varakis JN et al. Detection of $\mathrm{JC}$ virus DNA sequences and expression of the viral regulatory protein T-antigen in tumors of the central nervous system. Cancer Res 2001; 61: 4287-4293.

33. Berger JR, Concha M. Progressive multifocal leukoencephalopathy: the evolution of a disease once considered rare. J Neurovirol 1995; 1: 5-18.
34. Slamon DJ, Godolphin W, Jones LA, Holt JA, Wong SG, Keith DE et al. Studies of the HER-2/neu proto-oncogene in human breast and ovarian cancer. Science 1989; 244 : 707-712.

35. Bal MM, Das Radotra B, Srinivasan R, Sharma SC. Expression of c-erbB-4 in medulloblastoma and its correlation with prognosis. Histopathology 2006; 49: 92-93.

36. D'Ambrosio C, Keller SR, Morrione A, Lienhard GE, Baserga R, Surmacz E. Transforming potential of the insulin receptor substrate 1. Cell Growth Differ 1995; 6: 557-562.

37. Drakas R, Tu X, Baserga R. Control of cell size through phosphorylation of upstream binding factor 1 by nuclear phosphatidylinositol 3-kinase. Proc Natl Acad Sci USA 2004; 101: 9272-9276.

38. Baker J, Liu JP, Robertson EJ, Efstratiadis A. Role of insulin-like growth factors in embryonic and postnatal growth. Cell 1993; 75: 73-82.

39. Reiss K, Cheng W, Ferber A, Kajstura J, Li P, Li B et al. Overexpression of insulin-like growth factor- 1 in the heart is coupled with myocyte proliferation in transgenic mice. Proc Natl Acad Sci USA 1996; 93: 8630-8635.

40. Singec I, Knoth R, Meyer RP, Maciaczyk J, Volk B, Nikkhah G et al. Defining the actual sensitivity and specificity of the neurosphere assay in stem cell biology. Nat Methods 2006; 3: 801-806. 